\title{
Correlation of Metabolic Factors with Endometrial Atypical Hyperplasia and Endometrial Cancer: Development and Assessment of a New Predictive Nomogram
}

\author{
He Zhang (iD \\ Weimin Kong \\ Chao Han \\ Tingting Liu \\ Jing Li (ID \\ Dan Song
}

Department of Gynecological Oncology, Beijing Obstetrics and Gynecology Hospital, Beijing Maternal and Child Health Care Hospital, Capital Medical University, Beijing, People's Republic of China
Correspondence: Weimin Kong Department of Gynecological Oncology, Beijing Obstetrics and Gynecology Hospital, Beijing Maternal and Child Health Care Hospital, Capital Medical University, Qihelou Street, Dongcheng District, Beijing, 100006, People's

Republic of China

Tel +86 I880I273620

Email kwm1967@ccmu.edu.cn
Purpose: This study aimed to investigate the association of metabolic factors with endometrial atypical hyperplasia and endometrial cancer, and to develop a nomogram model to predict the risk of developing endometrial cancer.

Patients and Methods: We collected data of patients with endometrial atypical hyperplasia and endometrial cancer as the case group and then selected patients with simple hyperplasia or polypoid hyperplasia of the endometrium during the same period as the control group using the age-matched method. Laboratory results of metabolic factors were retrieved from the clinical data of the two groups of patients. Multivariable logistic regression analysis was used to determine the risk factors associated with endometrial malignant hyperplasia and to develop a nomogram prediction model of risk factors associated with endometrial malignant hyperplasia. Discrimination, calibration, and clinical usefulness of the prediction model were assessed using the $\mathrm{C}$-index, calibration plot, and decision curve analysis.

Results: Predictors included in the column line graph model were age, hypertension, diabetes, BMI, uric acid, and hyperlipidemia. We calculated the $\mathrm{C}$-index of the model and performed bootstrap validation. Our nomogram model had good discriminatory power and was well calibrated. Decision curve analysis was also used to guide the practical application of this column line graph model.

Conclusion: The development of endometrial malignant hyperplasia is significantly associated with metabolic factors: BMI $>25$, hyperuricemia, and hyperlipidemia are the main risk factors. Hypertension, hyperglycemia and elevated CA199 were also associated with the development of endometrial malignant hyperplasia. The nomogram prediction model based on physical examination and laboratory testing developed in this study can be used as a rapid method for predicting the risk of endometrial malignancy development and screening for risk factors in a population of women with metabolism-related high-risk factors.

Keywords: risk factors, gynecologic oncology, predictors, nomogram

\section{Introduction}

Endometrial cancer is one of the most common gynecological malignancies. The latest cancer statistics from the American Cancer Society show that in 2020 the number of new cases of endometrial cancer in the United States was 65,620, and the number of deaths was 12,590 . The incidence of malignant endometrial tumors in women ranked fourth, and the incidence of death from endometrial cancer ranked 
sixth. ${ }^{1}$ Endometrial atypical hyperplasia (EAH) is a precancerous lesion of endometrial cancer. ${ }^{2}$ The mechanisms of endometrial cancer and endometrial atypical hyperplasia pathological process are complex and polycentric in time and space. In recent years, the development of early diagnostic surgery and radiotherapy has improved patient outcomes to a great extent. However, the diagnosis and treatment of endometrial lesions at an early stage, surgery to preserve reproductive function, and research into the treatment of advanced and recurrent patients remain promising.

Metabolic syndrome (MS) is defined as a complex of metabolic risk factors associated with a variety of diseases, including cardiovascular disease and diabetes. ${ }^{3}$ The main components of metabolic syndrome are obesity, diabetes or impaired glucose tolerance, dyslipidemia, and hypertension. Furthermore, metabolic syndrome includes insulin resistance, hyperuricemia, and microalbuminuria. Recent studies have found that the incidence of endometrial cancer is increasing with the rise in the prevalence of metabolic diseases (eg, obesity, hypertension, and diabetes). ${ }^{4}$ To this end, we collected case data from the Department of Gynecological Oncology, Beijing Obstetrics and Gynecology Hospital, Capital Medical University, to retrospectively analyze metabolic parameters in patients with endometrial hyperplasia and malignant lesions, evaluate relevant metabolic risk factors for the development of endometrial malignant hyperplasia, and establish a nomogram prediction model to predict the risk of endometrial malignant hyperplasia.

\section{Patients and Methods}

\section{Patients}

Research approval was obtained from Beijing Obstetrics and Gynecology Hospital, Capital Medical University's Ethics Committee (approval no 2021-KY-050-01). A total of 205 patients with 102 cases of endometrial atypical hyperplasia and 103 cases of endometrial carcinoma admitted to the Department of Gynecological Oncology, Beijing Obstetrics and Gynecology Hospital, Capital Medical University, from January 1, 2010 to December 31, 2015, were collected as the study group. Considering that changes in metabolic parameters such as hypertension may be related to age or atypicality, we paired patients in the study group 1:1 with those in the control group. The control group was randomly selected from a cohort of patients suffering from simple hyperplasia or polypoid hyperplasia of the endometrium during the same period. The age difference of patients between each pairing was no more than 3 years. All patients were diagnosed with endometrioid adenocarcinoma, endometrial atypical hyperplasia, simple hyperplasia, or polypoid hyperplasia. They voluntarily signed informed consent for the study before registration, had good compliance, and were willing to closely cooperate with relevant examinations and follow-up. Patients with tumors in combination with other sites or who developed distant metastases were excluded. Patients using oral contraceptives or hormone therapy, taking hypoglycemic or hypotensive medications, or suffering from other metabolic disorders such as thyroid disease were excluded because of their potential impact on metabolic levels. We also excluded patients with incomplete laboratory results or poor compliance.

After taking a full and complete medical history and performing a physical examination, the patients underwent hysteroscopic localization of endometrial biopsy. Indications for endometrial biopsy include abnormal uterine bleeding, endometrial thickening, and cavity occupancy. ${ }^{5,6}$ Before the endometrial biopsy, transvaginal ultrasound was used to measure the thickness of the patient's endometrium. After sampling, the specimens were placed in formalin and histopathological examination was performed.

\section{Histopathological Analysis}

Patients were divided into two groups based on the results of endometrial biopsies. The case group (205 cases) was diagnosed with endometrial atypical hyperplasia (102 cases) or endometrial carcinoma (103 cases). The control group (205 cases) was diagnosed with endometrial simple hyperplasia or polypoid hyperplasia. The endometrium in both the secretory and proliferative phases, atrophic endometrium, endometrial polyps, and epithelial fragments containing mucus-like material were considered normal endometrium.

\section{Clinical and Biochemical Measurements}

Metabolic syndrome was diagnosed under the WHO definition, when the participants presented with diabetes or impaired fasting glycemia or impaired glucose tolerance or insulin resistance, and two or more of five risk determinants: obesity (BMI $>30$ or waist-to-hip ratio $>0.85$ ), dyslipidemia (triglycerides $\geq 1.7 \mathrm{mmol} / \mathrm{L}$ or HDL cholesterol $<1.0 \mathrm{mmol} / \mathrm{L}$ ), and hypertension (blood pressure $>$ 
140/90 $\mathrm{mmHg}$ ). In addition, we used the ATP III cut-off values for total cholesterol (240 mg/dL for TC) and lowdensity lipoprotein cholesterol (160 mg/dL for LDL), above which levels are considered high. Body mass index (BMI) was calculated as weight in kilograms divided by the square of height in meters, and overweight was defined as a BMI of $\geq 25$.

\section{Statistical Analysis}

All statistical analyses were carried out using $\mathrm{R}$ software. (v.4.0.2; https://www.R-project.org). Normally distributed variables were tested using independent samples $t$-test. The $\chi^{2}$ test was used for categorical variables. Single-factor and multifactor logistic regression analysis was used to calculate the odds ratio (OR) and its $95 \%$ confidence interval (CI) to estimate the correlation effect and correlation between each factor and endometrial pathology, and to establish a nomogram prediction model. $\mathrm{P}<0.05$ risk factors and some recognized risk factors associated with lesions were included in this model. All $\mathrm{P}$-values are two-tailed and $\mathrm{P}<0.05$ was considered statistically significant.

\section{Results}

\section{Patients' Characteristics}

A total of 410 patients with postoperative pathological findings confirming endometrioid adenocarcinoma, endometrial atypical hyperplasia, benign endometrial hyperplasia, or polypoid hyperplasia were included in this study. Patients included 205 cases $(50.0 \%)$ of endometrioid adenocarcinoma and endometrial atypical hyperplasia, and 205 cases $(50.0 \%)$ of benign endometrial hyperplasia or polypoid hyperplasia. The mean age of the study group was 49.4 years; there were 150 cases $(73.2 \%)$ of irregular vaginal bleeding, 85 cases $(41.5 \%)$ of hypertension, 69 cases $(33.7 \%)$ of elevated fasting glucose and/or diabetes, 97 cases (47.3\%) of dyslipidemia, and 59 cases (28.8\%) of metabolic syndrome. The control group had a mean age of 48.9 years, 77 cases of irregular vaginal bleeding (37.6\%), 44 cases of hypertension (21.5\%), 22 cases of elevated fasting glucose and/or diabetes (10.73\%), 39 cases of dyslipidemia (19.02\%), and 10 cases of metabolic syndrome (4.88\%). Other basic conditions and metabolic parameters between patient groups are shown in Table 1.

\section{Feature Selection}

Based on a retrospective analysis of the statistical characteristics of disease and treatment in 410 patients, six potential predictors were selected and generalized from 13 predictors associated with metabolic factors by comparing metabolicrelated data in the case and control groups by single-factor logistic regression analysis. These potential predictors included hypertension, diabetes mellitus, BMI, hyperlipidemia, hyperuricemia, and CA199 (Table 2).

The results of multifactorial logistic regression analysis for hypertension, hyperglycemia, BMI $>25$, hyperlipidemia, hyperuricemia, and CA199 are shown in Table 3. The BMI > 25 differences, hyperglycemia, hyperlipidemia, hyperuricemia, and CA199 between the study and control groups were statistically significant $(\mathrm{P}<0.05)$. Another risk factor with a P-value close to 0.05 or recognized in clinical and guideline settings, namely hypertension, was also considered as a metabolic risk factor associated with malignant endometrial hyperplasia in the present retrospective analysis. The above independent predictors were included in the nomogram model (Figure 1).

In this study, we constructed and validated the above nomogram model based on six predictors, blood pressure, blood glucose, blood lipids, BMI, uric acid, and CA199. These variables were selected based on the results of logistic regression analysis and risk factors highly associated with the risk of developing endometrial malignant hyperplasia as reported in previous studies. Patients can be scored for basic conditions such as metabolic indicators, and the scores are summed to obtain a total score that gives the corresponding predicted probability of developing endometrial malignant hyperplasia. The higher the score, the higher the probability of endometrial malignant hyperplasia. Therefore, clinicians should be mindful of making an early identification of the high-risk group for endometrial malignant hyperplasia from the perspective of glucose and lipid metabolism. They can then develop standard methods for diagnosis and symptom assessment of high-risk patients to provide early diagnosis and intervention, such as controlling blood glucose, reasonable weight loss, and regular health monitoring, and to educate patients about symptoms and regular follow-up.

\section{Apparent Performance of the Risk Factors Associated with Endometrial Malignant Hyperplasia in the Retrospective Analysis}

The calibration curves of the nomogram model for predicting metabolic factors and risk of endometrial malignant hyperplasia showed good agreement in this retrospective analysis (Figure 2). The C-index for 
Table I Baseline Characteristics of Patients

\begin{tabular}{|c|c|c|c|c|}
\hline \multirow[t]{2}{*}{ Characteristics } & \multicolumn{4}{|l|}{ n (\%) } \\
\hline & Study Group $(n=205)$ & Control Group $(n=205)$ & Total $(n=4 \mid 0)$ & $\mathbf{P}$ \\
\hline \multicolumn{5}{|l|}{ Age } \\
\hline$\leq 50$ & $103(50.24)$ & $82(78.85)$ & $185(59.87)$ & \\
\hline $50-60$ & $72(35.12)$ & $19(18.27)$ & 91 (29.45) & \\
\hline$>60$ & $30(14.63)$ & $3(2.88)$ & $33(10.68)$ & \\
\hline$($ mean $\pm S D)$ & $49.42 \pm 10.69$ & $48.87 \pm 10.60$ & $49.15 \pm 10.64$ & 0.75 \\
\hline \multicolumn{5}{|l|}{$A \cup B$} \\
\hline Yes & $150(73.17)$ & 77 (37.56) & $227(55.37)$ & \\
\hline No & $55(26.83)$ & $128(62.44)$ & $183(44.63)$ & $<0.001$ \\
\hline \multicolumn{5}{|l|}{ Menstrual Status } \\
\hline Menopause & $83(40.49)$ & $46(22.44)$ & $186(45.37)$ & \\
\hline Menstruating & $122(59.5 \mid)$ & $159(77.56)$ & $224(54.63)$ & $<0.001$ \\
\hline \multicolumn{5}{|c|}{ Metabolic Characteristics } \\
\hline \multicolumn{5}{|l|}{$\operatorname{BMI}\left(\mathrm{kg} / \mathrm{m}^{2}\right)$} \\
\hline Normal(BMI<25) & $76(37.07)$ & 147 (7I.7I) & $223(54.39)$ & \\
\hline Overweight (BMI $\geq 25$ ) & $129(62.93)$ & 58 (28.29) & $187(45.61)$ & $<0.001$ \\
\hline \multicolumn{5}{|l|}{$\mathrm{SBP}(\mathrm{mmHg})$} \\
\hline$<140$ & $127(61.95)$ & $159(77.56)$ & $286(69.76)$ & \\
\hline$\geq 140$ & $78(38.05)$ & $46(22.44)$ & $124(30.24)$ & 0.001 \\
\hline \multicolumn{5}{|l|}{$\mathrm{DBP}(\mathrm{mmHg})$} \\
\hline$<90$ & $123(60.00)$ & 179 (87.32) & $302(73.66)$ & \\
\hline$\geq 90$ & $82(40.00)$ & $26(12.68)$ & $108(26.34)$ & $<0.001$ \\
\hline \multicolumn{5}{|l|}{$\mathrm{HBP}$} \\
\hline Yes & $85(4 I .46)$ & $44(21.46)$ & $129(31.46)$ & \\
\hline No & 120 (58.54) & 161 (78.54) & $28 I(68.54)$ & $<0.001$ \\
\hline \multicolumn{5}{|l|}{$\mathrm{CHO}(\mathrm{mmol} / \mathrm{L})$} \\
\hline $0-5.2$ & $110(53.66)$ & 154 (75.12) & $264(64.39)$ & \\
\hline$>5.2$ & $95(46.34)$ & $51(24.88)$ & $146(35.61)$ & $<0.001$ \\
\hline \multicolumn{5}{|l|}{ GLU (mmol/L) } \\
\hline$\geq 5.5$ & $69(33.66)$ & $22(10.73)$ & $91(22.20)$ & \\
\hline$<5.5$ & $136(66.34)$ & $183(89.27)$ & $319(77.80)$ & $<0.001$ \\
\hline \multicolumn{5}{|l|}{ CEA (ug/L) } \\
\hline $0-5$ & 203 (99.02) & 203 (99.02) & 406 (99.02) & \\
\hline$>5$ & $2(0.98)$ & $2(0.98)$ & $4(0.98)$ & $>0.05$ \\
\hline \multicolumn{5}{|l|}{ CAI $25(\mathrm{U} / \mathrm{mL})$} \\
\hline $0-30.2$ & $172(83.90)$ & $192(93.66)$ & $364(88.78)$ & \\
\hline$>30.2$ & $33(16.10)$ & $13(6.34)$ & 46 (II.22) & 0.002 \\
\hline \multicolumn{5}{|l|}{ CAI99 (U/mL) } \\
\hline $0-30.9$ & $162(79.02)$ & $194(94.63)$ & $356(86.83)$ & \\
\hline$>30.9$ & $43(20.98)$ & II (5.37) & $54(13.17)$ & $<0.001$ \\
\hline \multicolumn{5}{|l|}{ TG (mmol/L) } \\
\hline $0-1.7$ & 134 (65.37) & $180(87.80)$ & 314 (76.59) & \\
\hline$>1.7$ & 7I (34.63) & $25(12.20)$ & $96(23.4 I)$ & $<0.001$ \\
\hline
\end{tabular}

(Continued) 
Table I (Continued).

\begin{tabular}{|c|c|c|c|c|}
\hline \multirow[t]{2}{*}{ Characteristics } & \multicolumn{4}{|l|}{ n (\%) } \\
\hline & Study Group $(n=205)$ & Control Group $(n=205)$ & Total $(n=4 \mid 0)$ & $\mathbf{P}$ \\
\hline \multicolumn{5}{|l|}{$\mathrm{HDL}(\mathrm{mmol} / \mathrm{L})$} \\
\hline $1.04-1.60$ & $138(67.32)$ & $173(81.73)$ & $311(75.85)$ & \\
\hline$<1.04$ & $67(32.68)$ & $32(18.27)$ & $99(24.15)$ & $<0.001$ \\
\hline \multicolumn{5}{|l|}{ LDL (mmol/L) } \\
\hline $2.07-3.37$ & $134(65.37)$ & I7I (83.4I) & 305 (74.39) & \\
\hline$>3.37$ & 7I (34.63) & 34 (16.59) & $105(25.61)$ & $<0.001$ \\
\hline \multicolumn{5}{|l|}{$\mathrm{UA}(\mu \mathrm{mol} / \mathrm{L})$} \\
\hline $155-357$ & $165(80.49)$ & $194(94.63)$ & $359(87.56)$ & \\
\hline$>357$ & $40(19.5 I)$ & II (5.37) & $51(12.44)$ & $<0.001$ \\
\hline \multicolumn{5}{|l|}{ HLP } \\
\hline Yes & $97(47.32)$ & $39(19.02)$ & $136(33.17)$ & \\
\hline No & $108(52.68)$ & $166(80.98)$ & $274(66.83)$ & $<0.001$ \\
\hline \multicolumn{5}{|l|}{ MS } \\
\hline Yes & $59(28.78)$ & $10(4.88)$ & $69(16.83)$ & \\
\hline No & 146 (7I.22) & $195(95.12)$ & $34 \mid(83.17)$ & $<0.001$ \\
\hline
\end{tabular}

Note: $P<0.05$ (statistically significant).

Abbreviations: AUB, abnormal uterine bleeding; BMI, body mass index; SBP, systolic blood pressure; DBP, diastolic blood pressure; HBP, high blood pressure; CHO, total cholesterol; GLU, glucose; CEA, carcinoma embryonic antigen; TG, triglyceride; HDL, high-density lipoprotein; LDL, low-density lipoprotein; UA, uric acid; HLP, hyperlipidemia; MS, metabolic syndrome.

predicting the risk of developing metabolic factors and endometrial malignancy was 0.782 (95\% CI: 0.738 0.826). The Receiver Operating Characteristic (ROC) curve for the nomogram prediction model is shown in Figure 3. Area under the curve $(\mathrm{AUC})=0.7816538$.

Table 2 Single-Factor Logistic Regression of Metabolic FactorRelated Predictors

\begin{tabular}{|l|l|l|l|}
\hline Variables & $\boldsymbol{\beta}$ & Odds Ratio (95\% CI) & $\mathbf{P}$ \\
\hline Age & -0.029 & $0.971(0.945-0.998)$ & $0.037^{*}$ \\
SBP & -0.022 & $0.978(0.957-0.999)$ & $0.039^{*}$ \\
DBP & 0.080 & $1.083(1.046-1.122)$ & $<0.00 I^{*}$ \\
FBG & 0.563 & $1.756(1.216-2.535)$ & $0.003^{*}$ \\
BMI & 0.103 & $1.108(1.028-1.194)$ & $0.007^{*}$ \\
CEA & 0.150 & $1.162(0.857-1.575)$ & 0.335 \\
CAI25 & 0.005 & $1.005(0.997-1.013)$ & 0.248 \\
CAI99 & 0.032 & $1.033(1.012-1.054)$ & $0.002^{*}$ \\
CHO & 1.517 & $4.557(1.882-11.038)$ & $<0.00 I^{*}$ \\
TG & -0.315 & $0.730(0.454-1.174)$ & 0.195 \\
HDL & -1.528 & $0.217(0.062-0.759)$ & $0.017^{*}$ \\
LDL & -1.121 & $0.326(0.133-0.798)$ & $0.014^{*}$ \\
UA & 0.006 & $1.006(1.002-1.010)$ & $0.002^{*}$ \\
\hline
\end{tabular}

Note: $\beta$ is the regression coefficient. $* P<0.05$ (statistically significant). Abbreviations: SBP, systolic blood pressure; DBP, diastolic blood pressure; FBG, fasting blood-glucose; BMI, body mass index; CEA, carcinoma embryonic antigen; TG, triglyceride; HDL, high-density lipoprotein; LDL, low-density lipoprotein; UA, uric acid.
Figure 4 shows the ROC curves for each independent metabolic factor endpoint. The corrected C-index of 0.771 was obtained after the bootstrapping validation of the model, which demonstrated that the model had a good predictive ability.

\section{Clinical Use}

The decision curve analysis of the nomogram model for predicting the risk of developing metabolic factors and

Table 3 Prediction Factors for Endometrial Malignant Hyperplasia

\begin{tabular}{|l|l|l|l|}
\hline $\begin{array}{l}\text { Intercept and } \\
\text { Variable }\end{array}$ & $\begin{array}{l}\text { Prediction } \\
\text { Model }\end{array}$ & & \\
\hline & $\boldsymbol{\beta}$ & Odds Ratio (95\% CI) & $\mathbf{P}$ \\
\hline HBP & 0.4594 & $1.583(0.952-2.632)$ & 0.076 \\
HGlu & 0.7711 & $2.162(1.190-3.997)$ & 0.012 \\
BMI>25 & 0.9069 & $2.477(1.546-3.975)$ & $<0.001$ \\
HUA & 1.0277 & $2.795(1.330-6.252)$ & 0.008 \\
HLP & 1.0204 & $2.774(1.705-4.555)$ & $<0.001$ \\
CAI99 & 1.4731 & $4.363(2.120-9.634)$ & $<0.001$ \\
\hline
\end{tabular}

Note: $\beta$ is the regression coefficient.

Abbreviations: HBP, high blood pressure; HGlu, hyperglycemia; BMl, body mass index; HUA, hyperuricemia; HLP, hyperlipidemia; CA199, Carbohydrate antigen 199. 
Points

$\begin{array}{llllllllll}0 & 10 & 20 & 30 & 40 & 50 & 60 & 70 & 80 & 90\end{array}$

HUA

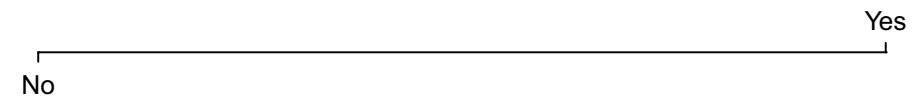

HBP

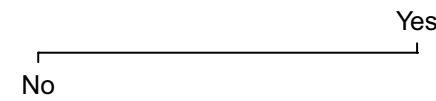

BMI

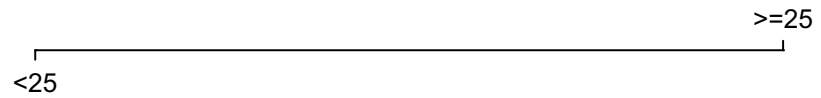

HLP

HGlu

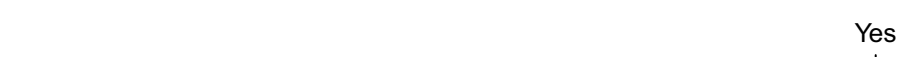

Yes

No

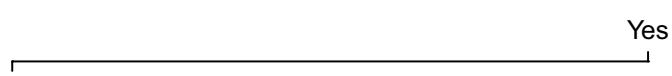

No

$>30.9$

CA199

$0-30.9$

Total Points

\begin{tabular}{llllllllll}
\hline 0 & 50 & 100 & 150 & 200 & 250 & 300 & 350 & 400
\end{tabular}

Risk of endometrial malignant hyperplasia

$\begin{array}{rllllll}0.3 & 0.4 & 0.5 & 0.6 & 0.7 & 0.8 & 0.9\end{array}$

Figure I Nomogram prediction model for the risk of developing endometrial malignant hyperplasia.

Notes: The endometrial malignant hyperplasia nomogram prediction models were developed in this retrospective analysis, with HUA, HBP, BMI, HLP, HGlu, CAI99.

Abbreviations: HUA, hyperuricemia; HBP, hypertension; BMI:, mass index; HLP, hyperlipidemia; HGlu, hyperglycemia; CAI99, Carbohydrate antigen 199.

endometrial malignant hyperplasia is shown in Figure 5. The decision curves show that when the threshold probability is between $22 \%$ and $86 \%$, using this nomogram line graph to predict the risk of developing metabolic factors and endometrial malignant hyperplasia, respectively, provides more benefit to the patient.

\section{Discussion}

Nomograms are now widely used in oncology and clinical medicine as prediction and analysis tools for risk factors and prognosis. Nomograms rely on a user-friendly and easy-to-learn interface that can improve the accuracy of predictions and help clinicians make better clinical decisions. Our study is the first to apply nomograms to risk factors related to gynecologic cancer and glycolipid metabolism, using six clinically accessible variables related to disease and treatment that are capable of initially predicting the risk of endometrial malignant hyperplasia in a metabolically abnormal population. This study provides a relatively accurate tool for predicting endometrial malignancy in women with metabolic disorders and contributes to the individualized prediction of patients' risk of developing endometrial malignant hyperplasia. Intra-group validation of the retrospective analysis showed good discrimination and calibration. In particular, there was still a high $\mathrm{C}$-index when the model was validated by bootstrapping, indicating that the retrospective analysis had a large sample size and was widely used, generalizable, and accurate in practice.

Metabolic syndrome is a complex disorder in which insulin resistance, hyperinsulinemia, impaired glucose tolerance, type 2 diabetes mellitus, dyslipidemia, and visceral obesity are a series of risk factors associated with the development of metabolic syndrome. ${ }^{3}$ Endometrial cancer is one of the cancers most closely associated with metabolic diseases, and the incidence of endometrial cancer is increasing as the incidence of metabolic diseases increases. ${ }^{7}$ Endometrial atypical hyperplasia is also 


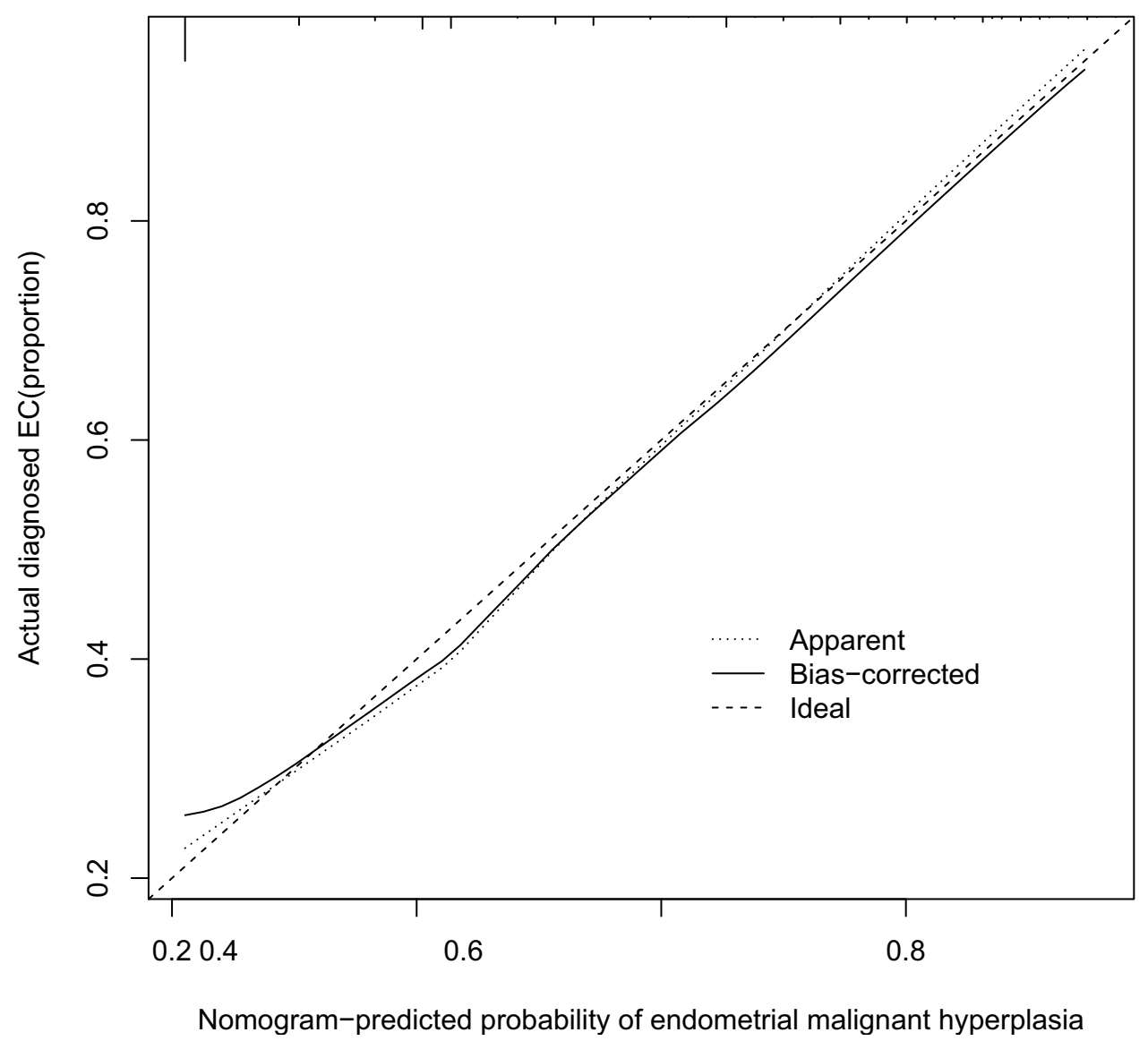

Figure 2 The nomogram prediction model of this retrospective analysis of calibration curves.

Notes: The $\mathrm{x}$-axis indicates the predicted endometrial malignant hyperplasia risk. The $y$-axis indicates the actual diagnosed endometrial malignant hyperplasia risk. The diagonal dotted line represents aperfect prediction by an ideal model. The solid line represents the performance of the nomogram, of which acloser fit to the diagonal dotted line represents abetter prediction.

strongly associated with metabolic factors. ${ }^{8}$ A metaanalysis by Esposito et al showed that metabolic syndrome is strongly associated with an increased risk of endometrial cancer. ${ }^{9}$ Kitson et al reported that women diagnosed with endometrial cancer have a higher incidence of metabolic abnormalities than women without endometrial cancer. ${ }^{10}$ In our retrospective analysis, age, hypertension, diabetes mellitus, BMI, hyperlipidemia, and blood uric acid were associated with the development of endometrial malignancy in patients. Of these, obesity, diabetes, and hypertension are commonly referred to as the metabolic triad of endometrial cancer. In recent years, several studies have shown that metabolic syndrome caused by obesity, diabetes, and hypertension is closely associated with the incidence and poor prognosis of endometrial cancer. It has been shown that patients with hyperglycemia, hyperlipidemia, and hypertension are twice as likely to develop endometrial cancer as normal people. ${ }^{11}$ The aim of this study was to investigate the association of metabolic factors with endometrial atypical hyperplasia and endometrial cancer. This study is also the first to identify a potential link between high uric acid and endometrial malignancy. The exact mechanisms by which metabolic syndrome affects the development of endometrial lesions are complex and may be related to the following factors.

\section{Obesity and Endometrial Cancer}

Adipose tissue is an important endocrine organ that secretes a variety of hormones including leptin and lipofuscin, as well as chemokines that modulate tumor behavior, inflammation, and the tumor microenvironment. ${ }^{12}$ The excessive accumulation of adipose tissue in obese patients leads to increased levels of free fatty acids in the circulatory system and increased expression of serum adipokines (eg, leptin, endolipoproteins, and cytokines), which ultimately leads to insulin resistance. Among these, decreased serum adiponectin levels and increased chronic inflammation in obese patients are important 


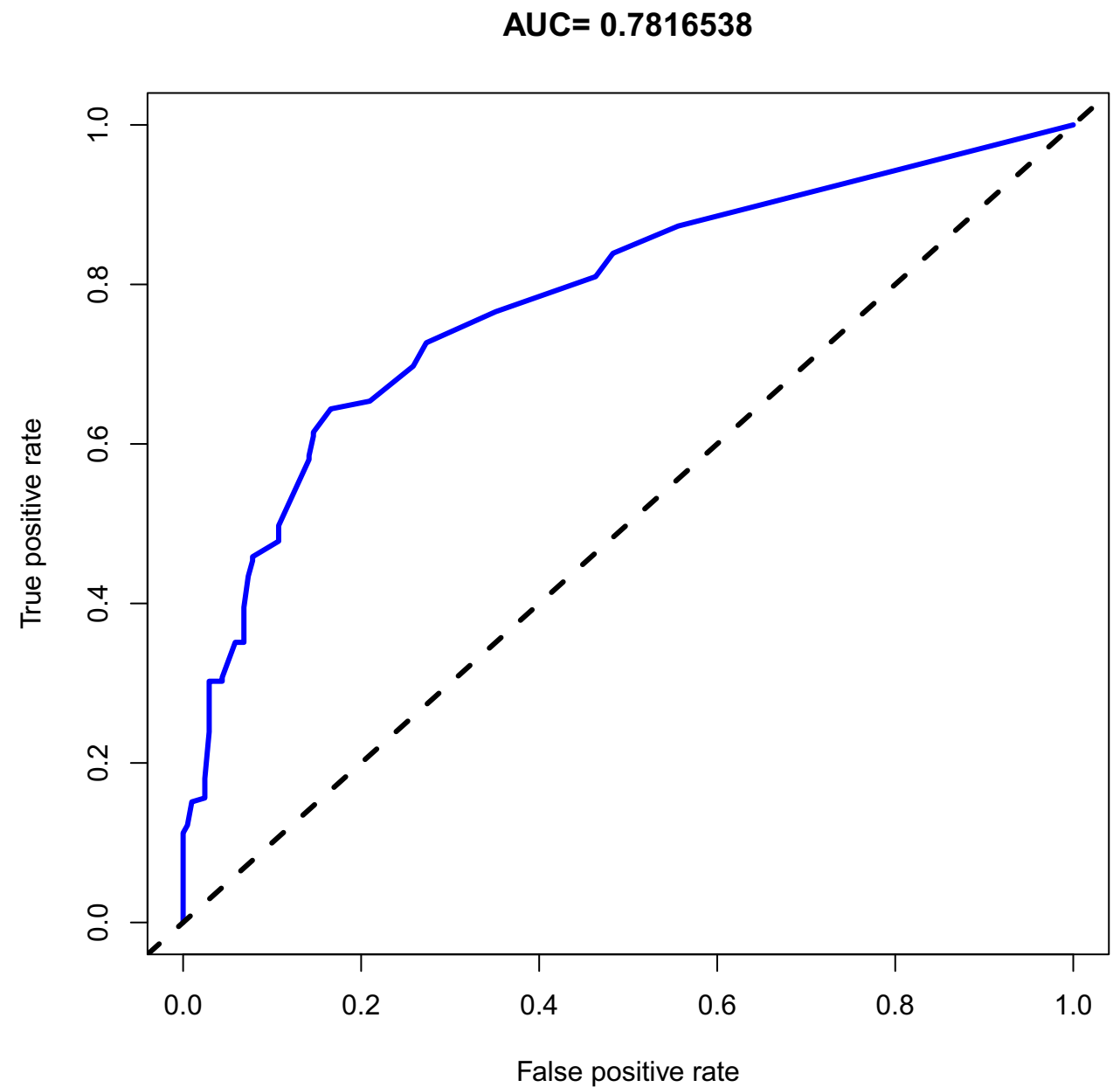

Figure 3 ROC curves for the nomogram predictive model.

Notes: The vertical axis indicates the true positive rate (TPR) and the horizontal axis indicates the false positive rate (FPR). The area under the curve AUC $=0.7816538$.

factors that increase the risk of endometrial cancer. Overexpansion of fat in obese patients leads to adipose dysfunction and inflammation, thereby increasing the levels of pro-inflammatory factors throughout the body. ${ }^{13}$ Chronic inflammation also lays the groundwork for the cancer development band. This inflammatory response contributes to the increased prevalence and mortality of endometrial cancer associated with obesity. Obesity may also increase the risk of endometrial cancer by indirectly affecting estrogen levels. ${ }^{14}$ Obesity-induced insulin resistance leads to hyperinsulinemia, which may reduce the synthesis of sex hormone-binding protein (SHBG) by increasing the bioavailability of insulin-like growth factor-1 (IGF-1), resulting in elevated estrogen levels. Adipose tissuederived aromatase also converts androstenedione to estradiol, resulting in elevated serum estradiol levels, estradiol binding to estrogen receptors, and ultimately, transcription factor recruitment, and gene transcription may be activated or inhibited. ${ }^{12,15}$ Ward et al reported that people with a history of bariatric surgery and those who were able to maintain a normal weight after surgery were able to reduce their risk of uterine malignancy by $71 \%$ and $81 \%$, respectively. ${ }^{16}$ These studies show a close relationship between obesity and the development of endometrial cancer.

\section{Hyperglycemia and Endometrial Cancer}

Diabetes mellitus is a risk factor for endometrial cancer. Patients with type 2 diabetes mellitus are often associated with hyperinsulinemia and insulin resistance. In a state of insulin resistance, elevated insulin levels directly or indirectly influence the development of endometrial cancer. ${ }^{17}$ Direct mechanisms include the activation of key signaling pathways such as PI3K/Akt, Ras/MAPK; and the interaction of signaling pathways between insulin, IGF-1, and estrogen. Among the indirect mechanisms, excess insulin 


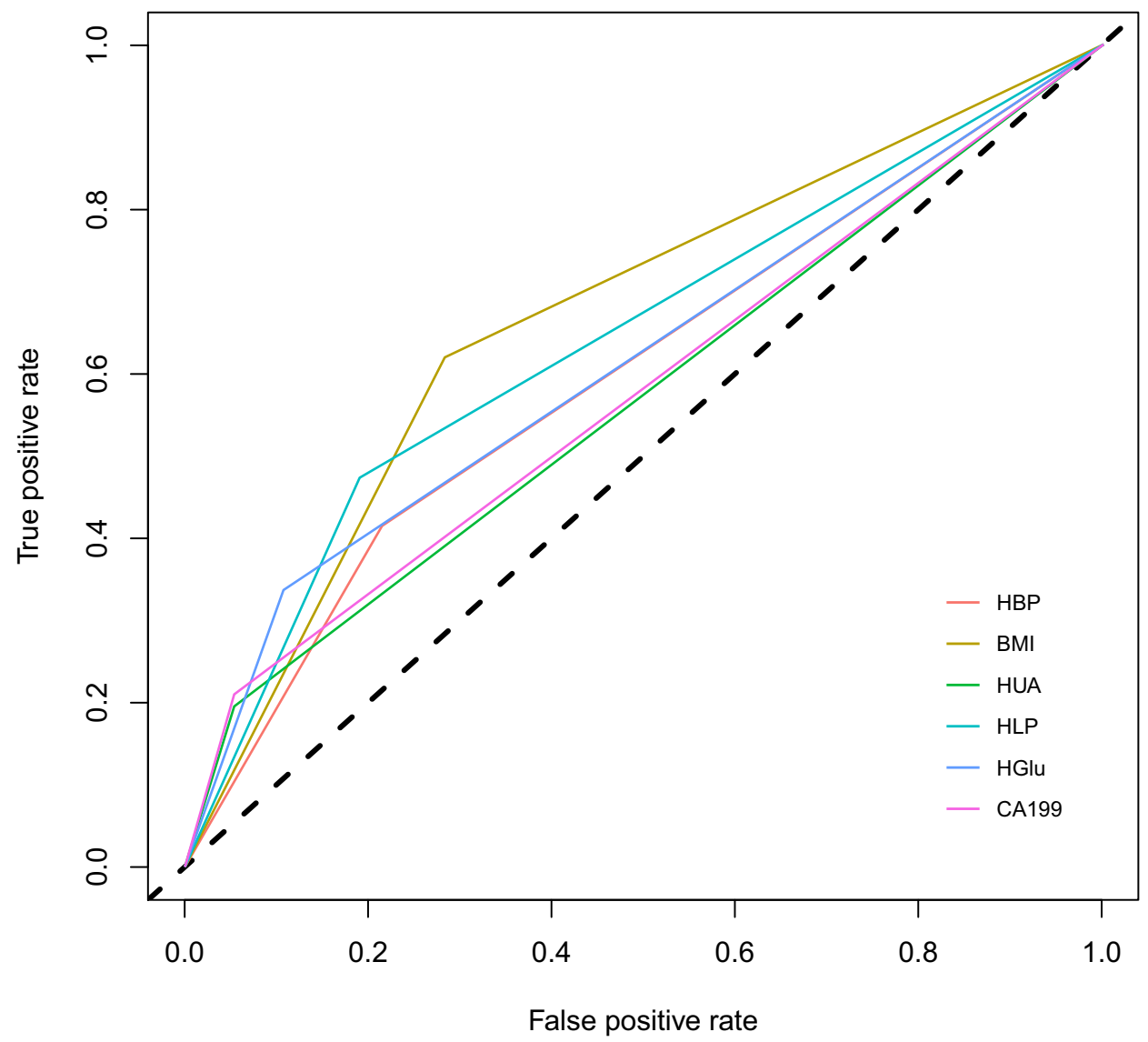

Figure 4 The ROC curves for each independent metabolic factor endpoint.

Notes: The vertical axis indicates the true positive rate (TPR) and the horizontal axis indicates the false positive rate (FPR).

leads to decreased blood levels of SHBG and increased blood levels of estrogen and androgen, thereby promoting the development of endometrial cancer. ${ }^{18}$ It has been shown that serum insulin levels can increase the incidence of endometrial cancer in a dose-dependent manner. Moreover, insulin resistance is a key pathophysiological process in obesity, diabetes, hypertension, and even metabolic syndrome. In addition, diabetic patients in a high glucose state can promote the proliferation and invasion of endometrial cancer cells by regulating the expression of ER $\alpha /$ Glut4, which promotes the expression of vascular endothelial growth factor/vascular endothelial growth factor receptor. ${ }^{19}$ In addition, high glucose also increases glucose uptake and glycolytic activity by modulating AMPK/mTOR/S6 and MAPK signaling pathways, thereby increasing the invasiveness of endometrial cancer cells. ${ }^{20}$

Most of the epidemiological evidence also suggests that diabetes is a risk factor for the development of endometrial lesions. A meta-analysis of 16 studies by Friberg et al showed that diabetes was statistically significantly associated with an increased risk of endometrial cancer (RR 2.10; 95\% CI 1.75-2.53). ${ }^{21}$ Diabetes not only increases the incidence of endometrial cancer but also increases the relative risk of death in endometrial cancer patients. Epidemiological studies have shown that the risk of endometrial cancer is 2.12 times higher in diabetic patients and 2.45 times higher in overweight patients than in normal patients. ${ }^{22}$ Type 2 diabetes combined with obesity significantly increases the risk of endometrial cancer. ${ }^{17}$

\section{Dyslipidemia and Endometrial Cancer}

There are more studies on the relationship between endometrial cancer and obesity and diabetes mellitus, but fewer studies on the relationship between endometrial cancer and hypertension and dyslipidemia. Abnormal lipid metabolism, especially elevated free fatty acids, is strongly associated with the progression of endometrial cancer. ${ }^{23}$ The possible mechanisms of dyslipidemia associated with endometrial cancer risk are: activation of the fatty acid 


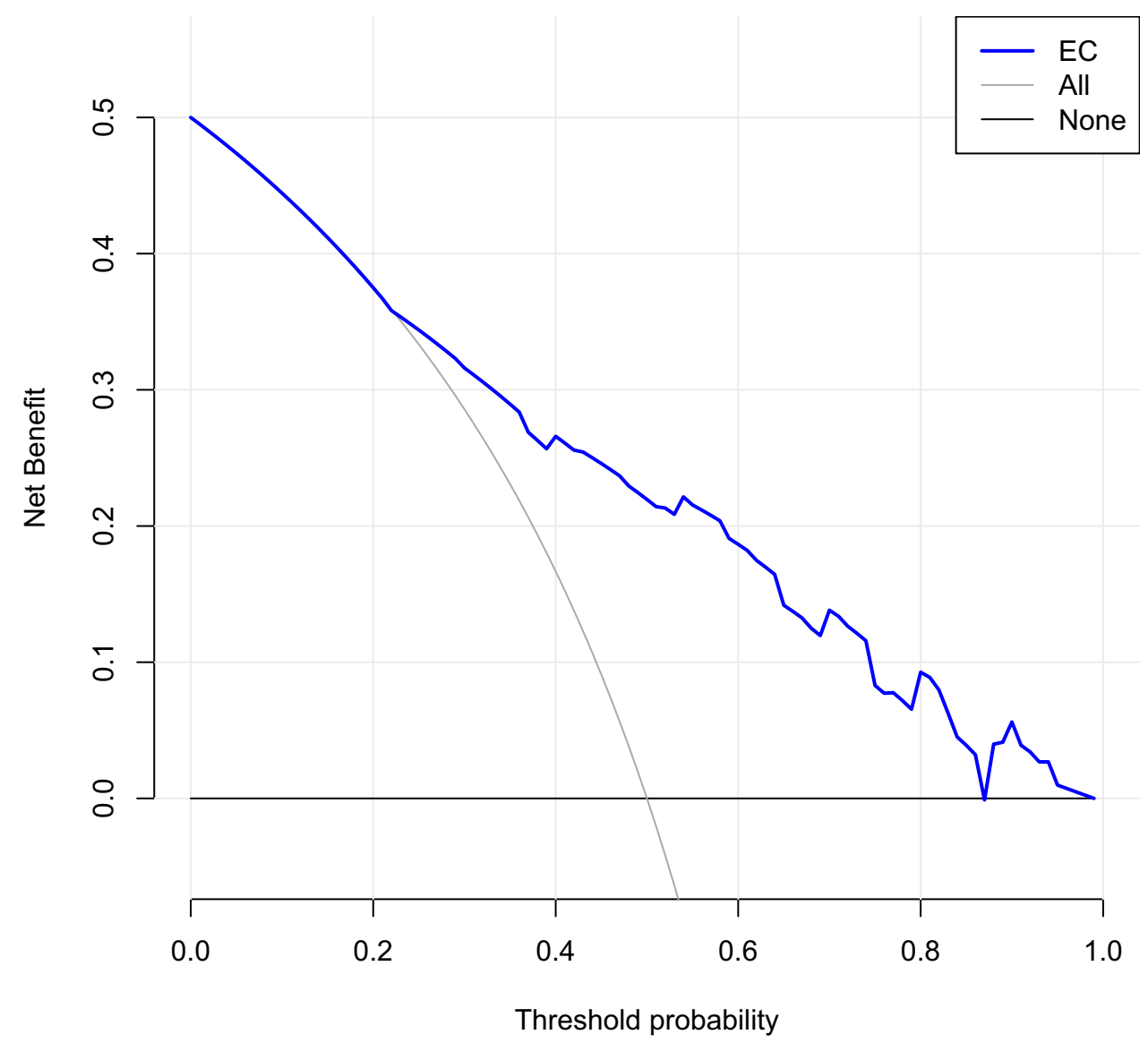

Figure 5 Decision curve analysis for the nomogram prediction model.

Notes: The $y$-axis measures the net benefit. The dotted line represents the endometrial malignant hyperplasia risk nomogram. The thin solid line represents the hypothesis that all patients do not receive the intervention. Athick solid line indicates that no patient has arelevant metabolic factor disease. The decision curves show that it is meaningful to use this nomogram to predict patients' risk of morbidity and intervene on treatment when the threshold probability is within $22-86 \%$.

and aminoglycan pathways leading to the production of reactive oxygen species (ROS) in mitochondria, causing cellular oxidative stress; or excessive accumulation of ROS clusters, which interact with intracellular lipids, proteins, and DNA, leading to altered cell membrane and enzyme function, induction of cellular damage, and ultimately tumorigenesis. ${ }^{24}$ In addition, serum cholesterol is elevated in obese patients, and cholesterol activates the transcriptional activity of endometrial cancer cells through an ER-dependent pathway, which promotes the proliferation of endometrial cancer cells. ${ }^{25}$

\section{Other Relevant Doctrines and Endometrial Cancer}

A meta-analysis by Aune et al shows that hypertension is a risk factor for endometrial cancer. ${ }^{26}$ A large cohort study of 290,000 women found that the risk of endometrial cancer increased with increasing blood pressure levels, particularly systolic blood pressure levels. ${ }^{27}$ However, because hypertension is a condition that is closely related to lifestyle factors and a number of medical conditions (including diet, BMI, physical activity, and diabetes), there are still definite limitations to the current study. The biological mechanism by which hypertension increases the risk of endometrial cancer is unclear and may be related to cellular senescence and apoptosis inhibition caused by chronic hypertension.

Serum uric acid (SUA) is an antioxidant that is abundant in the blood and plays an important role as a free radical scavenger, and has been linked to many lifestylerelated diseases such as metabolic syndrome and type 2 diabetes. $^{28,29}$ Numerous in vitro and animal studies suggest that the relationship between SUA metabolism and oxidative stress may be involved in the onset and/or development of disease. Epidemiological investigations have shown that individuals with higher uric acid levels may have a higher incidence of cancer. A large prospective study of 28,613 older women showed that elevated uric 
acid $(>5.41 \mathrm{mg} / \mathrm{dL})$ was independently associated with an increased risk of death from all cancer types. ${ }^{30,31}$

In addition, dynamic interactions between cells and their microenvironment play an important role in regulating the growth of normal tissues and cancer cells. ${ }^{32}$ The tumor cell microenvironment includes tumor cells and other cells, such as fibroblasts, lymphocytes, macrophages, adipocytes, and other secretory factors. An abnormal imbalance in the tumor cell microenvironment often leads to tumorigenesis. This also suggests that metabolic syndrome and its associated signaling molecules can not only act directly on tumor cells but also further remodel the tumor immune microenvironment, thereby accelerating the progression of endometrial cancer. ${ }^{33}$

\section{Limitations}

Our current study had some limitations. First, this retrospective analysis does not represent all Chinese patients with endometrial lesions. Patients with no access to treatment were also excluded from this retrospective analysis. Second, the risk factors included in this study did not include all the potential risk factors that influence the development of metabolic factors and endometrial lesions. Third, although the nomogram model for this retrospective analysis has been extensively tested by bootstrapping, it has not been possible to externally validate this model and there is uncertainty about its generalizability to other endometrial lesion populations in other regions and countries. It needs to be externally evaluated in a broader endometrial lesion population.

\section{Conclusion}

Our study revealed the correlation between hypertension, hyperglycemia, BMI $>25$, hyperlipidemia, hyperuricemia, CA199, and endometrial malignant hyperplasia based on the retrospective analysis of metabolic-related factors in 410 patients. Among them, CA199 is a tumor marker closely related to endometrial cancer. ${ }^{34}$ Hypertension, diabetes mellitus, BMI $>25$, hyperlipidemia, hyperuricemia, and high CA199 index are all high-risk factors for endometrial malignant hyperplasia, and the probability of this disease is greatly increased in patients with multiple highrisk factors. This study also established a new nomogram model with good accuracy to develop an effective tool for predicting the risk of endometrial malignancy in women with metabolic-related risk factors. Previous scientific studies have provided effective therapeutic targets for the treatment of endometrial cancer based on the relationship between metabolic syndrome and cancer, but lifestyle improvement and early intervention remain the most important components of preventing morbidity and mortality from metabolic syndrome-associated endometrial cancer. This model provides clinicians with the necessary assistance in identifying and predicting a patient's risk of developing endometrial malignant growth at an early stage based on their metabolic profile. By estimating the risk of the disease, clinicians and patients can take more adequate and necessary measures in terms of lifestyle monitoring and medical interventions. In addition, the model can be used as a tool for screening patients at high risk for endometrial lesions in clinical studies. For example, the model may be used to select reliable patients with good adherence to medications in prospective controlled studies. When conducting retrospective studies, we could also exclude patients with poor adherence, thus making the analysis more reliable. This nomogram model still needs to be externally validated to broaden the predictive power and applicability. In the meantime, further studies will be conducted to determine whether individual interventions based on the nomogram model can reduce the risk of endometrial malignant hyperplasia in women with metabolism-related risk factors.

\section{Ethical Approval and Informed Consent}

All procedures performed in studies involving human participants were in accordance with the ethical standards of the Science and Technology Division of Beijing Obstetrics and Gynecology Hospital, Capital Medical University and with the 1964 Helsinki Declaration and its later amendments or comparable ethical standards.

\section{Informed Consent}

Informed consent was obtained from all individual participants included in the study.

\section{Acknowledgments}

We appreciate the effort of the physicians for enrolling patients and thank all the patients involved for allowing us to analyze their clinical data.

\section{Author Contributions}

All authors made a significant contribution to the work reported, whether that was in the conception, study design, execution, acquisition of data, analysis and interpretation, or in all the following areas: took part in drafting, revising 
or critically reviewing the article; gave final approval of the version to be published; have agreed on the journal to which the article has been submitted; and agree to be accountable for all aspects of the work.

\section{Disclosure}

The authors declare that they have no conflicts of interest.

\section{References}

1. Siegel RL, Miller KD, Jemal A. Cancer statistics, 2020. CA Cancer $J$ Clin. 2020;70(1):7-30. doi:10.3322/caac.21590

2. Sanderson PA, Critchley HO, Williams AR, et al. New concepts for an old problem: the diagnosis of endometrial hyperplasia. Hum Reprod Update. 2017;23(2):232-254.

3. Eckel RH, Grundy SM, Zimmet PZ. The metabolic syndrome. Lancet. 2005;365(9468):1415-1428. doi:10.1016/S0140-6736(05) 66378-7

4. Shou H, Yan K, Song J, et al. Metabolic syndrome affects the long-term survival of patients with non-endometrioid carcinoma of the uterine corpus. Int J Gynaecol Obstet. 2020;148(1):96-101. doi:10.1002/ijgo.12984

5. ACOG Committee Opinion. The use of hysteroscopy for the diagnosis and treatment of intrauterine pathology. Obstet Gynecol. 2020;135(3):e138-e148. doi:10.1097/AOG.0000000000003712

6. Yen $\mathrm{CF}$, Chou $\mathrm{HH}, \mathrm{Wu} \mathrm{HM}$, et al. Effectiveness and appropriateness in the application of office hysteroscopy. J Formos Med Assoc. 2019;118(11):1480-1487. doi:10.1016/j.jfma.2018.12.012

7. Morice P, Leary A, Creutzberg C, et al. Endometrial cancer. Lancet. 2016;387(10023):1094-1108. doi:10.1016/S0140-6736(15)00130-0

8. Raffone A, Travaglino A, Saccone G, et al. Diabetes mellitus is associated with occult cancer in endometrial hyperplasia. Pathol Oncol Res. 2020;26(3):1377-1384. doi:10.1007/s12253-019-00684-3

9. Esposito K, Chiodini P, Colao A, et al. Metabolic syndrome and risk of cancer: a systematic review and meta-analysis. Diabetes Care. 2012;35(11):2402-2411. doi:10.2337/dc12-0336

10. Kitson SJ, Lindsay J, Sivalingam VN, et al. The unrecognized burden of cardiovascular risk factors in women newly diagnosed with endometrial cancer: a prospective case control study. Gynecol Oncol. 2018;148(1):154-160. doi:10.1016/j.ygyno.2017.11.019

11. Arthur RS, Kabat GC, Kim MY, et al. Metabolic syndrome and risk of endometrial cancer in postmenopausal women: a prospective study. Cancer Causes Control. 2019;30(4):355-363. doi:10.1007/ s10552-019-01139-5

12. Deng T, Lyon CJ, Bergin S, Caligiuri MA, Hsueh WA. Obesity, inflammation, and cancer. Annu Rev Pathol. 2016;11:421-449. doi:10.1146/annurev-pathol-012615-044359

13. Secord AA, Hasselblad V, Von Gruenigen VE, et al. Body mass index and mortality in endometrial cancer: a systematic review and meta-analysis. Gynecol Oncol. 2016;140(1):184-190. doi:10.1016/j. ygyno.2015.10.020

14. Sung H, Siegel RL, Torre LA, et al. Global patterns in excess body weight and the associated cancer burden. CA Cancer J Clin. 2019;69 (2):88-112.

15. Roque DR, Makowski L, Chen TH, et al. Association between differential gene expression and body mass index among endometrial cancers from The Cancer Genome Atlas Project. Gynecol Oncol. 2016;142(2):317-322. doi:10.1016/j.ygyno.2016.06.006
16. Ward KK, Roncancio AM, Shah NR, et al. Bariatric surgery decreases the risk of uterine malignancy. Gynecol Oncol. 2014;133 (1):63-66. doi:10.1016/j.ygyno.2013.11.012

17. Lai Y, Sun C. Association of abnormal glucose metabolism and insulin resistance in patients with atypical and typical endometrial cancer. Oncol Lett. 2018;15(2):2173-2178.

18. Mu N, Zhu Y, Wang Y, et al. Insulin resistance: a significant risk factor of endometrial cancer. Gynecol Oncol. 2012;125(3):751-757. doi:10.1016/j.ygyno.2012.03.032

19. Gu CJ, Xie F, Zhang B, et al. High glucose promotes epithelial-mesenchymal transition of uterus endometrial cancer cells by increasing ER/GLUT4-mediated VEGF secretion. Cell Physiol Biochem. 2018;50(2):706-720. doi:10.1159/000494237

20. Wallbillich JJ, Josyula S, Saini U, et al. High glucose-mediated STAT3 activation in endometrial cancer Is inhibited by metformin: therapeutic implications for endometrial cancer. PLoS One. 2017;12 (1):e0170318. doi:10.1371/journal.pone.0170318

21. Friberg E, Orsini N, Mantzoros CS, et al. Diabetes mellitus and risk of endometrial cancer: a meta-analysis. Diabetologia. 2007;50 (7):1365-1374. doi:10.1007/s00125-007-0681-5

22. Yang X, Wang J. The role of metabolic syndrome in endometrial cancer: a review. Front Oncol. 2019;9:744. doi:10.3389/ fonc. 2019.00744

23. Madak-Erdogan Z, Band S, Zhao YC, et al. Free fatty acids rewire cancer metabolism in obesity-associated breast cancer via estrogen receptor and mTOR signaling. Cancer Res. 2019;79(10):2494-2510.

24. Schmandt RE, Iglesias DA, Co NN, et al. Understanding obesity and endometrial cancer risk: opportunities for prevention. Am J Obstet Gynecol. 2011;205(6):518-525. doi:10.1016/j.ajog.2011.05.042

25. Gibson DA, Collins F, Cousins FL, et al. The impact of 27-hydroxycholesterol on endometrial cancer proliferation. Endocr Relat Cancer. 2018;25(4):381-391. doi:10.1530/ERC-17-0449

26. Aune D, Sen A, Vatten LJ. Hypertension and the risk of endometrial cancer: a systematic review and meta-analysis of case-control and cohort studies. Sci Rep. 2017;7:44808.

27. Bjørge T, Stocks T, Lukanova A, et al. Metabolic syndrome and endometrial carcinoma. Am J Epidemiol. 2010;171(8):892-902. doi:10.1093/aje/kwq006

28. Dovell F, Boffetta P. Serum uric acid and cancer mortality and incidence: a systematic review and meta-analysis. Eur $J$ Cancer Prev. 2018;27(4):399-405. doi:10.1097/CEJ.0000000000000440

29. Yang J, Wang Y, Zhao Q, et al. Association of serum uric acid with increased risk of cancer among hypertensive Chinese. Int J Cancer. 2017;141(1):112-120. doi:10.1002/ijc.30731

30. Kühn T, Sookthai D, Graf ME, et al. Albumin, bilirubin, uric acid and cancer risk: results from a prospective population-based study. $\mathrm{Br}$ J Cancer. 2017;117(10):1572-1579. doi:10.1038/bjc.2017.313

31. Yiu A, Van Hemelrijck M, Garmo H, et al. Circulating uric acid levels and subsequent development of cancer in 493,281 individuals: findings from the AMORIS study. Oncotarget. 2017;8 (26):42332-42342. doi:10.18632/oncotarget.16198

32. DeBerardinis RJ, Phimister EG. Tumor microenvironment, metabolism, and immunotherapy. $N$ Engl J Med. 2020;382(9):869-871. doi:10.1056/NEJMcibr1914890

33. Iyengar NM, Gucalp A, Dannenberg AJ, et al. Obesity and cancer mechanisms: tumor microenvironment and inflammation. J Clin Oncol. 2016;34(35):4270-4276. doi:10.1200/JCO.2016.67.4283

34. Hutt S, Tailor A, Ellis P, et al. The role of biomarkers in endometrial cancer and hyperplasia: a literature review. Acta Oncol. 2019;58 (3):342-352. doi:10.1080/0284186X.2018.1540886 


\section{Publish your work in this journal}

Cancer Management and Research is an international, peer-reviewed open access journal focusing on cancer research and the optimal use of preventative and integrated treatment interventions to achieve improved outcomes, enhanced survival and quality of life for the cancer patient.
The manuscript management system is completely online and includes a very quick and fair peer-review system, which is all easy to use. Visit http://www.dovepress.com/testimonials.php to read real quotes from published authors.

Submit your manuscript here: https://www.dovepress.com/cancer-management-and-research-journal 